investigated for cystic fibrosis $(17.9 \%$ vs. $4.9 \% \mathrm{p}=0.007)$. They were significantly more likely to have grown a pseudomonas, staph aureus or a gram negative bacteria in their sputum in the last 12 months $(29.4 \%$ vs. $5.4 \%, \mathrm{p}=0.0036)$ and be treated with either oral or nebulised long term antibiotics $(35.0 \%$ vs. $12.5 \%, \mathrm{p}=0.012$ ) graph 1 .

Conclusions Patients attending specialist bronchiectasis clinics were more likely to be managed according to BTS quality standards. Specialist non-CF bronchiectasis clinics may improve quality of care. Further longitudinal studies are needed to investigate if specialist clinics improve clinical outcomes.

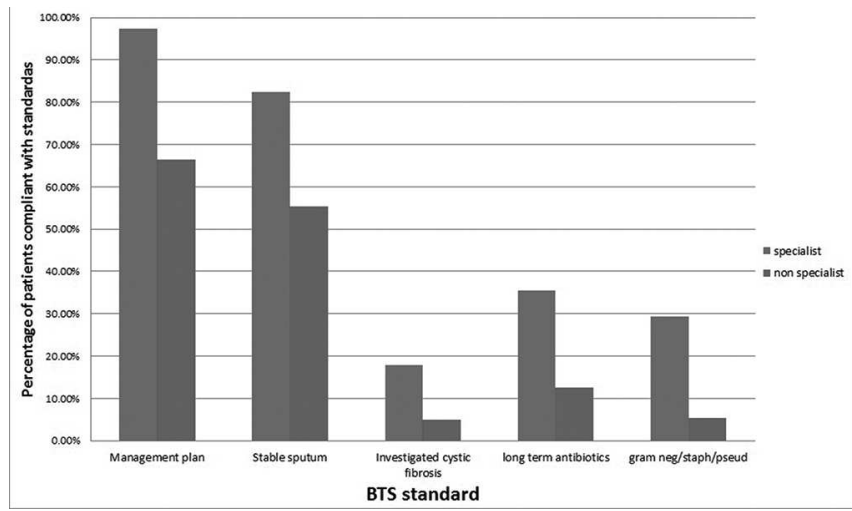

Abstract P109 Figure1 Comparison between specialist and non specialist clinics for management of bronchiectasis

\section{P110 DOES PREVIOUS EXACERBATION HISTORY PREDICT FUTURE EXACERBATIONS IN NON-CF BRONCHIECTASIS?}

${ }^{1}$ WS Salih, ${ }^{2}$ LP Poppelwell, ${ }^{1}$ RS Stretton, 'TCF Fardon, ' 1 JDC Chalmers; 'Department of

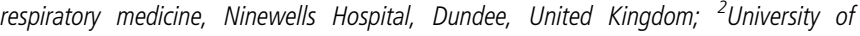
Dundee, Ninewells hospital, Dundee, UK

\subsection{6/thoraxjn-2013-204457.260}

Introduction The British Thoracic Society bronchiectasis guidelines recommend consideration of long term antibiotic therapy in patients with 3 or more exacerbations per year. A major goal of treatment in bronchiectasis is to reduce future exacerbation risk. It is not known how reliably a past history of frequent exacerbations predicts future exacerbations in bronchiectasis.

Methods Consecutive patients with non-CF bronchiectasis attending a specialist clinic in Tayside 2011-2012 were included. Patients commencing long term antibiotic therapy were excluded. Exacerbation frequency was obtained from patient histories verified against electronic antibiotic prescription data. Hospital admissions for severe exacerbations were recorded and the ability of prior exacerbation history to predict future hospital admissions assessed using the area under the receiver operator characteristic curve (AUC).

Results 90 patients with bronchiectasis were included. 54\% were female with a median age of 67 years. The majority had idiopathic bronchiectasis (58\%), followed by allergic bronchopulmonary aspergillosis (13.3\%), post-infective bronchiectasis $(8 \%)$ and connective tissue disease (5\%). The median FEV1 was 74\% (51-94) predicted.

In the first year of review, patients had a mean of 2.9 exacerbations (standard deviation 2.4), while in the second year, the mean number of exacerbations was 2.1 (standard deviation 1.9), $\mathrm{p}=0.02$.

Patients with a history of 3 exacerbations in year 1 had a higher frequency of exacerbations in year 2 (2.5 vs 1.8, $\mathrm{p}=0.03)$. The sensitivity of year 1 exacerbations to predict 3 or more exacerbations in year 2 was $52 \%$ (95\% confidence interval $31.9-71.3 \%)$ with a specificity of $64 \%(50.4-75.3 \%)$

Figure 1 shows the relationships between year 1 and year 2 exacerbation frequencies.

In a linear regression model, additional predictors of exacerbation in year 2 were $\mathrm{FEV}_{1} \%$ predicted and chronic bacterial colonisation, independent of previous exacerbation frequency.

24 patients were hospitalised for severe exacerbations in year 2. Prior exacerbation frequency was only a modest predictor of future hospitalisation risk, AUC 0.72 (95\% CI 0.61-0.83), p < 0.0001). (Figure 1)

Conclusion Prior history of exacerbations predicts future exacerbations and risk of severe exacerbations, but large variations in annual exacerbation frequency are observed. Other factors may need to be considered to more accurately identify patients at risk of future exacerbations and hospital admissions.

\section{Year 1}

Year 2

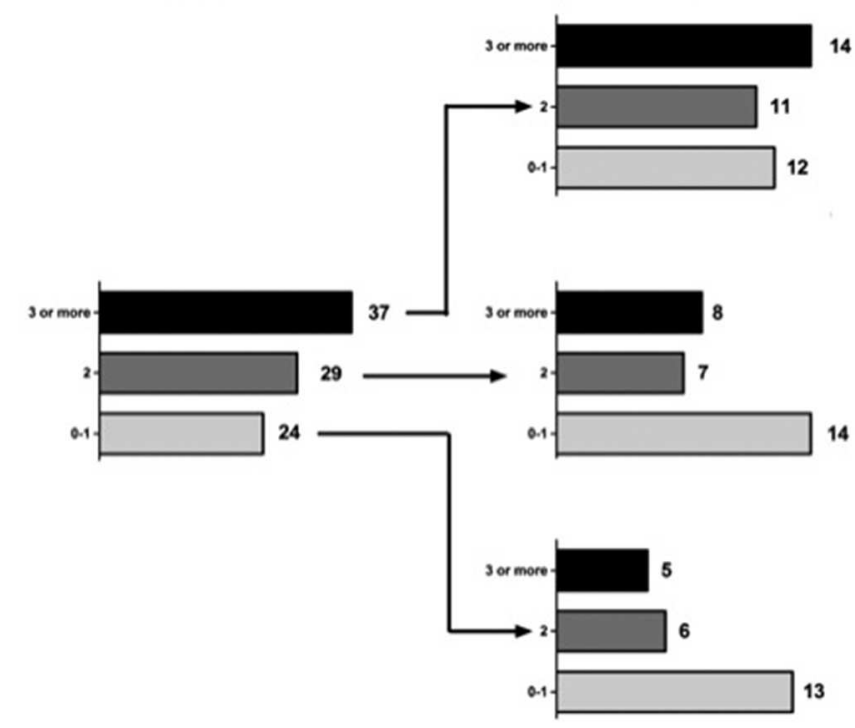

Abstract P110 Figure 1.

\section{P111 AZITHROMYCIN PRESCRIPTIONS IN A TEACHING HOSPITAL-DO WE NEED TO MONITOR FOR ADVERSE EFFECTS?}

P Rajagopalan, K De wit, J Barrett, R Breen; Guys and St Thomas Hospital NHS foundation Trust, London, United Kingdom

\subsection{6/thoraxjnl-2013-204457.261}

Introduction Azithromycin appears to have an important role in management of a number of conditions including non-CF bronchiectasis and COPD but with possible adverse effects including hearing loss and liver dysfunction that necessitate appropriate patient monitoring. We have examined our use of azithromycin and how we screen for complications in our Chest clinics.

Methods Data was collected on all azithromycin prescriptions provided at the Chest Clinic in a large UK teaching hospital over a 12 month period commencing 30-1-2012. In those patients receiving long-term azithromycin ( $\geq 12$ Months), we collected data on parameters including sputum microbiology, previous NTM, liver function tests (LFTs), audiology testing and Qtc interval recording. 
Results 192 prescriptions corresponding to 112 patients were identified. 62 of $112(56 \%)$ were prescribed long-term azithromycin. Bronchiectasis (60\%), COPD(19\%), asthma (8\%), ILD $(7 \%)$, Bronchiolitis and others $(6 \%)$ were the variety of diseases for which Azithromycin was prescribed. $28 \%$ had pseudomonas colonisation.

$51(46 \%)$ patients were prescribed long-term azithromycin. Of these, $21(25 \%)$ had been on azithromycin for less than 12 months.

Sixty eight (82\%) patients on long-term azithromycin had had LFTs and 3(3.5\%) had audiology testing in the preceding 12 months.21(25\%)had documented ECGs with Qtc interval.

No patients tested had hearing loss and no documented QTc prolongation.

The other common side effect noticed was GI upset in 6 patients (5.6\%).

The dosing was mostly $250 \mathrm{mg}$ three times a week $103(92 \%)$

There was also $500 \mathrm{mg}$ three times a week in $20(18 \%)$

H.influenzae (42\%)Staphylococcus aureus (21\%)Moraxella catarrhalis (11\%)coliform sp (10\%). We identified no new NTM in our Cohort.

Conclusion Although formal monitoring in this cohort was patchy, Significant documented adverse effects in this cohort were rare and optimal practice for long-term management of azithromycin use remains to be established.

\section{P112 DEEPER PHENOTYPING OF NON CF BRONCHIECTASIS THROUGH SPUTUM DIFFERENTIAL COUNTS}

${ }^{1} \mathrm{~J}$ Davison, ${ }^{1} \mathrm{G}$ Johnson, ${ }^{1} \mathrm{~K}$ Jiwa, ${ }^{1} \mathrm{~A}$ Cranson, ${ }^{1} \mathrm{R}$ Wake, ${ }^{1} \mathrm{~B}$ Hood, ${ }^{1} \mathrm{~T}$ Small, ${ }^{2} \mathrm{~J}$ Birch, ${ }^{2} \mathrm{~A}$ Fisher, ${ }^{2} \mathrm{~A}$ De Soyza; ${ }^{1}$ The Newcastle upon Tyne Hospitals NHS Foundation Trust, Newcastle upon Tyne, UK; ${ }^{2}$ Newcastle University, Newcastle upon Tyne, UK

\subsection{6/thoraxjnl-2013-204457.262}

Introduction and Objectives Non CF Bronchiectasis has diverse aetiologies. This includes idiopathic, systemic disease related and as a complication of asthma. Such diversity may be important in determining therapeutic strategies (personalised medicines) and may also be an important consideration in clinical trial design. This is increasingly relevant when neutrophil targeting or eosinophil targeted therapies are being developed. We hypothesised that patients could be phenotyped by sputum cytospins irrespective of suspected aetiology or disease severity.

Methods Patients underwent a standardised clinical phenotyping protocol including HRCT chest (Anwar et al 2013). Baseline therapy was recorded. Spontaneous sputa were collected in stable state and spirometry was undertaken according to guidelines. Sputum cell counts were calculated using standard methods with data expressed as medians and ranges.

Results Fifty three patients' data are reported. The M:F ratio was $1: 1.4$. The mean FEV1 predicted was $62 \%$, mean FEV1/VC ratio was $64 \%$. Forty three $(83 \%)$ were on inhaled corticosteroids and $24.5 \%$ had a historical diagnosis of asthma and /or ABPA. The predominant cell in sputa was neutrophils, median 94 (range 23-100\%), macrophages were the 2nd most prevalent cell type median 2.6 (range 0-75\%). Eosinophils showed a skewed distribution with median of 0.2 with a range of 0 $24.8 \%$. Four patients had sputum eosinophilia $>3 \%$. Of these, only 2 had a history of asthma and / or ABPA being diagnosed. Despite historical diagnoses of asthma and / or ABPA in 13 patients the eosinophil percentage was not statistically different to "non asthmatics". ( $\mathrm{P}=0.59 \mathrm{Chi} \mathrm{Sq}$ test $)$ This group included features of ABPA in 2 patients and significant atopy in another.
Conclusions Non CF bronchiectasis patients usually have sputum neutrophil dominance. Sputum eosinophilia is rare $(<10 \%$ of patients); however such patients may need alternative therapeutic strategies. Excluding bronchiectasis patients with a history of asthma from trials targeting neutrophils seems unnecessary. The neutrophilic predominant profiles in asthmatic bronchiectasis patients suggest either asthma misdiagnosis or that neutrophil predominant asthmatics may be more susceptible to developing bronchiectasis. Longitudinal studies are needed to determine if the sputum cell profiles are static in stable patients. These data may help develop a more personalised medicine approach in bronchiectasis.

\begin{tabular}{|c|c|c|c|c|}
\hline & NEUT & EOSIN & MACRO & LYMPH \\
\hline Median & 94.80 & 0.2 & 2.60 & 0.40 \\
\hline Max & 100.00 & 24.80 & 75.20 & 7.00 \\
\hline Min & 23.40 & 0.00 & 0.00 & 0.00 \\
\hline
\end{tabular}

\section{REFERENCES}

1. Anwar GA, et al. 2013 Phenotyping adults with non-cystic fibrosis bronchiectasis: A prospective observational cohort study. Respir Med. Jul;107(7):1001-7. doi: 10.1016/ j.rmed.2013.04.013. Epub 2013 May 11.

\section{P113 FREQUENT CO-DETECTION OF NON-TUBERCULOUS MYCOBACTERIA WITH OTHER MICROBES IN A UK CLINIC POPULATION: WHAT ARE THE IMPLICATIONS FOR TREATMENT?}

T Mahungu, M Yousefzadeh, N Shah, J Reading, J Hurst, S Burns, J Goldring, I Abubakar, I Cropley, S Hopkins, M Lipman; Royal Free London NHS Foundation Trust, London, United Kingdom

\subsection{6/thoraxjnl-2013-204457.263}

Introduction Non-Tuberculous Mycobacteria (NTM) are often isolated from patient samples, though their clinical relevance can be unclear. Treatment is not always effective and management decisions are usually based on repeat isolates with compatible clinical features. The presence of other micro-organisms, as well as the specific NTM itself, may be important. Here we report NTM and other microbe isolation frequency and their relationship to management decisions.

Methods All NTM samples isolated from liquid culture systems between $09 / 05 / 11$ and $03 / 04 / 13$ at our centre were identified using hospital pathology databases. Subject's negative mycobacterial cultures plus all positive relevant bacteria and virological isolates, as well as clinical history and progress were reviewed.

Results NTM were isolated on 257 occasions from 102 patients, who provided a total of 693 samples for mycobacterial culture. Adjusting for positive samples obtained within a month of each other, there were 170 isolates - 150 of which came from 90 patients' pulmonary samples. Common associated clinical conditions were non cystic fibrosis bronchiectasis $(28,31.1 \%)$, COPD (11, 12.2\%), and HIV infection (6, 6.7\%). The most frequent lung isolate was Mycobacterium avium intracellulare Complex, MAC, (47.8\%), followed by M. fortuitum(14.4\%), M. gordonae (10\%), and M. kansasii (8.9\%). Seven (7.8\%) patients had multiple NTM species identified. $40(44.4 \%)$ of the pulmonary patients also had bacteria or fungi isolated from lung samples. Pseudomonas sp. were present in 12 (13.3\%), Haemophilus influenzae in $10(11.1 \%)$, and Staphylococcus aureus in 6 (6.7\%) 